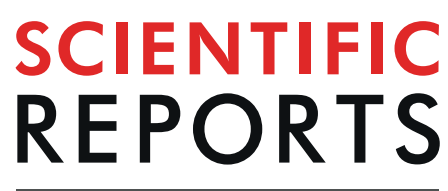

natureresearch

Received: 19 February 2019

Accepted: 22 August 2019

Published online: 18 September 2019

\section{The role of the GABAA receptor Alpha 1 subunit in the ventral hippocampus in stress resilience}

\author{
Z. Ardi ${ }^{1,2,3}$, A. Richter-Levin ${ }^{4}$, L. Xu $\mathbb{1}^{5,6,7}$, X. Cao ${ }^{8}$, H. Volkmer ${ }^{9}$, O. Stork ${ }^{10} \&$ \\ G. Richter-Levin (iD $1,4,11$
}

Pre-pubertal stress increases post-traumatic stress disorder (PTSD) susceptibility. We have previously demonstrated that enriched environment (EE) intervention immediately after pre-pubertal stress protects from the effects of trauma in adulthood. Here, we examined whether exposure to EE would also be beneficial if applied after exposure to trauma in adulthood. We have recently shown that exposure to juvenile stress and under-water trauma (UWT) is associated with increased expression of $\mathrm{GABA}_{\mathrm{A}}$ receptor subunit $\alpha 1$ in the ventral hippocampus. However, differentiating between affected and unaffected individuals, this increased expression was confined to stress-exposed, behaviorally unaffected individuals, suggesting upregulation of $\alpha 1$ expression as a potential mechanism of resilience. We now examined whether EE-induced resilience renders increased expression of $\alpha 1$ in the ventral hippocampus redundant when facing a trauma later in life. Adult rats were exposed to UWT, with pre-exposure to juvenile stress, and tested in the open field and elevated plus maze paradigms four weeks later. EE exposure during juvenility prevented pre-pubertal stress-induced vulnerability, but not if performed following UWT in adulthood. Furthermore, juvenile EE exposure prevented the trauma-associated increase in $\alpha 1$ expression levels. Our findings emphasize the importance of early interventions in order to reduce the likelihood of developing psychopathologies in adulthood.

Exposure to childhood stress is a well described risk factor of PTSD in adulthood ${ }^{1-3}$. Indeed, early adolescence (juvenility) constitutes a stress-sensitive period. Across species, including rats and humans, the juvenile brain is noticeably different from that of newborns, weanlings, or adults ${ }^{4,5}$. Accordingly, in rodents, we and others have demonstrated that juvenile (pre-pubertal) stress increases the risk for developing PTSD-related symptoms in adulthood $^{6-10}$. While attention is directed towards possible mechanisms of induced vulnerability, it is important to consider the possibility that some brain responses to stress exposure may be adaptive, aiming to reduce the risk for PTSD later in life. In line with that, we have recently demonstrated that pharmacological treatment with fluoxetine during juvenility reduced the risk of developing symptoms in an animal model of PTSD ${ }^{11}$. By contrast, the treatment was not effective when applied in adulthood ${ }^{9}$. We further demonstrated that juvenile animals exposed to an enriched environment (EE) starting immediately after the exposure to pre-pubertal stress and lasting into adulthood were protected from the deleterious effects of a trauma in adulthood ${ }^{12,13}$. To examine whether EE could be as effective in treating PTSD as it is in preventing its development, we now examined whether a similar

\footnotetext{
${ }^{1}$ Sagol Department of Neuroscience, University of Haifa, Haifa, 3498838, Israel. ${ }^{2}$ Present address: Department of Behavioral Sciences, Kinneret Academic College, Sea of Galilee, Tiberias, Israel. ${ }^{3}$ Sagol Center for Brain and Mind, Baruch Ivcher School of Psychology, Interdisciplinary Center (IDC), Herzliya, Israel. ${ }^{4}$ The Integrated Brain and Behavior Research Center (IBBR), University of Haifa, Haifa, 3498838, Israel. ${ }^{5}$ Key Laboratory of Animal Models and Human Disease Mechanisms, and Laboratory of Learning and Memory, Kunming Institute of Zoology, The Chinese Academy of Sciences, Kunming, 650223, China. ${ }^{6}$ CAS Centre for Excellence in Brain Science and Intelligent Technology, Shanghai, 200031, China. ${ }^{7}$ University of Chinese Academy of Sciences, Beijing, 100049, China. ${ }^{8}$ Key Laboratory of Brain Functional Genomics, MOE\&STCSM, East China Normal University, Shanghai, 200062, China. ${ }^{9}$ Deptartment Molecular Biology, Natural and Medical Sciences Institute at the University of Tübingen, Markwiesenstr. 55, 72770, Reutlingen, Germany. ${ }^{10}$ Department of Genetics \& Molecular Neurobiology, Institute of Biology, Otto-von-Guericke University Magdeburg, Magdeburg, 39120, Germany. ${ }^{11}$ Department of Psychology, University of Haifa, Haifa, 3498838, Israel. Correspondence and requests for materials should be addressed to G.R.-L. (email: galrichterlevin@gmail.com)
} 
exposure to EE could also be effective if given in adulthood, from immediately after the exposure to the adulthood trauma.

Many studies suggest that alterations in GABA functioning are associated with stress and trauma ${ }^{14-17}$. Recently, we have shown that exposing animals to under-water trauma (UWT) with pre-exposure to juvenile stress was associated with increased expression of $\mathrm{GABA}_{\mathrm{A}}$ receptor subunit $\alpha 1$, in the ventral but not dorsal hippocampus of exposed animals ${ }^{18}$. However, classification of the exposed population to affected and unaffected individuals by using behavioral profiling analysis revealed that the increased $\mathrm{GABA}_{\mathrm{A}}$ receptor $\alpha 1$ expression was evident only in exposed, unaffected animals, indicating a resilience-associated expression regulation ${ }^{18}$.

In this study, we therefore first examined whether the exposure to juvenile stress combined with UWT would be associated with a similar increased expression of $\alpha 1$ in the ventral hippocampus. We further examined whether such an increased expression of $\alpha 1$ would indeed be restricted only to exposed, unaffected individuals. Finally, we analyzed the effects of exposure to EE, either during juvenility or only in adulthood, on both behavior and $\alpha 1$ expression in the ventral hippocampus.

\section{Material and Methods}

Animals. Male Sprague-Dawley rats were purchased (Envigo Laboratories, Jerusalem, Israel) at postnatal day (PND) 22, weighing 30-50 g. Animals were housed in groups of 4-5 rats per cage, with ad libitum access to food and water $\left(22 \pm 2{ }^{\circ} \mathrm{C} ; 12: 12\right.$ hours light-dark cycle). All experiments were carried out in accordance with the NIH guide for care and use of laboratory animals. All experiments were approved by the ethics committee of the University of Haifa. All experimental procedures and assessments were preformed in designated rooms away from the vivarium between 9 AM and 3 PM.

Stress protocols. Juvenile stress (J) exposure. The current study followed the juvenile stress protocol as described before by Horovitz and colleagues ${ }^{8}$. Rats were exposed to three different stressors for three consecutive days (PND 27-29): Day 1, 10 minutes of forced swim stress; Day 2, elevated platform for $3 \times 30$ minutes (1-hour ITI in the home cage); Day 3, 2 hours in a restraint apparatus.

Odor and underwater trauma (UWT association learning). The protocol was performed as previously described by Ardi and colleagues ${ }^{18}$. In order to associate the odor with the UWT, all rats were first habituated to the "association cage" (a standard plastic cage covered with a plastic lid) for three days ( $2 \mathrm{~min}$ per day). On the 4 th day, following the $2 \mathrm{~min}$ habituation, all rats were exposed to vanilla odor $(100 \mu \mathrm{l}$ concentrated vanilla extract in $15 \mathrm{ml}$ of distilled water) for $30 \mathrm{~s}$ inside the cage. Immediately after odor exposure, all rats except controls were exposed to the UWT, by placing them in a water-filled plastic tank, and after $5 \mathrm{~s}$ of free swimming, restraining them under water for $45 \mathrm{~s}$ using a metal net. Control rats, on the 4th day, were exposed to the odor without exposure to UWT.

Enriched environment housing. Animals were housed together (4-5 rats per cage) in a large cage $\left(80 \times 80 \times 50 \mathrm{~cm}^{3}\right)$ and were provided with three differently shaped plastic containers, two tunnels, one colored platform, and two running wheels for four weeks. The objects and sawdust were changed twice a week.

Behavioral assessments. Behavioral assessments in the open field and elevated plus maze paradigms took place four weeks after UWT and odor (or only odor) exposure and were conducted as described before ${ }^{18}$.

Odor re-exposure. In order to change the original context of the odor reminder, the habituation cage that was used for the odor-UWT association learning was filled with a different type of sawdust than the type used during learning and the walls of the habituation cage were covered with geometric shapes. In the behavioral assessment room, rats were first habituated for $2 \mathrm{~min}$ and then were exposed for $30 \mathrm{~s}$ to the same vanilla odor that was used for association learning (equal odor concentration). Immediately following odor exposure, rats were subjected to behavioral tests.

Open field (OF) test. Rats were placed in the corner of the OF (under dim red-light illumination), and were left to explore the arena for $5 \min ^{6,18}$.

Elevated plus maze test $(E P M)^{6,18}$ was carried out 24 hours after the OF test. Rats were placed in the center of the maze (two opposing open arms/closed arms, under full light illumination), facing one of the open arms, and were left to explore the maze for $5 \mathrm{~min}$.

Rat behavior in the OF and EPM was recorded and then analyzed using EthoVision XT8 video tracking system (Noldus, Wageningen, Netherlands).

Experimental groups and design. The experimental design is depicted in Fig. 1. Following delivery and five days of acclimation period, rats were randomly assigned to the different experimental groups: (1) Control, $\mathrm{n}=19$; (2) Juvenile stress + UWT in adulthood (A) exposures ( $+\mathrm{A}, \mathrm{n}=16$ ); (3) Juvenile stress + UWT exposures + enriched environment housing in juvenility $(\mathrm{J}+\mathrm{EE}+\mathrm{A}, \mathrm{n}=16)$; (4) Juvenile stress + UWT exposures + enriched environment housing in adulthood $(J+A+E E, n=17)$. All rats except controls were exposed to juvenile stress (PND 27-29), and on the following day, J + EE + A rats were transferred from standard housing conditions to EE housing conditions (PND 30-62), while all other groups were subjected to normal housing conditions. In adulthood (PND 59-62), all rats except controls were exposed to the UWT protocol. Control rats were exposed to the same behavioral procedure without the UWT exposure. Immediately following the day of $\mathrm{UWT}, \mathrm{J}+\mathrm{EE}+\mathrm{A}$ rats returned to standard housing conditions and $\mathrm{J}+\mathrm{A}+\mathrm{EE}$ rats were subjected to EE conditions (PND 62-94). Four weeks after UWT (PND 93-94), rats were subjected to behavioral assessments in the open field and elevated plus maze. Immediately following the last behavioral assessment, rats were decapitated, and their brains were collected. 


\begin{tabular}{|c|c|c|c|c|c|}
\hline PND & $\underline{27-29}$ & $\underline{30-62}$ & $\underline{59-62}$ & $\underline{62-94}$ & 93-94 \\
\hline Group & Juv stress & EE(J) & UWT(t) & $\mathrm{EE}(\mathrm{A})$ & \multirow{5}{*}{ 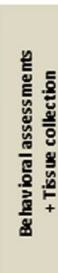 } \\
\hline Control & - & - & - & - & \\
\hline$J+A$ & + & - & + & - & \\
\hline $\mathrm{J}+\mathrm{EE}+\mathrm{A}$ & + & + & + & - & \\
\hline $\mathrm{J}+\mathrm{A}+\mathrm{EE}$ & + & - & + & + & \\
\hline
\end{tabular}

Figure 1. Experimental design. J + A rats were exposed to juvenile stress at PND 27-29 and to underwater trauma (UWT) and odor association learning at PND 59-62. J $+\mathrm{EE}+\mathrm{A}$ and $\mathrm{J}+\mathrm{A}+\mathrm{EE}$ rats were exposed to the same stressors but were housed in environmentally enriched cages either in juvenility or in adulthood. Control rats were not exposed to juvenile stress, UWT, or environmental enrichment conditions.
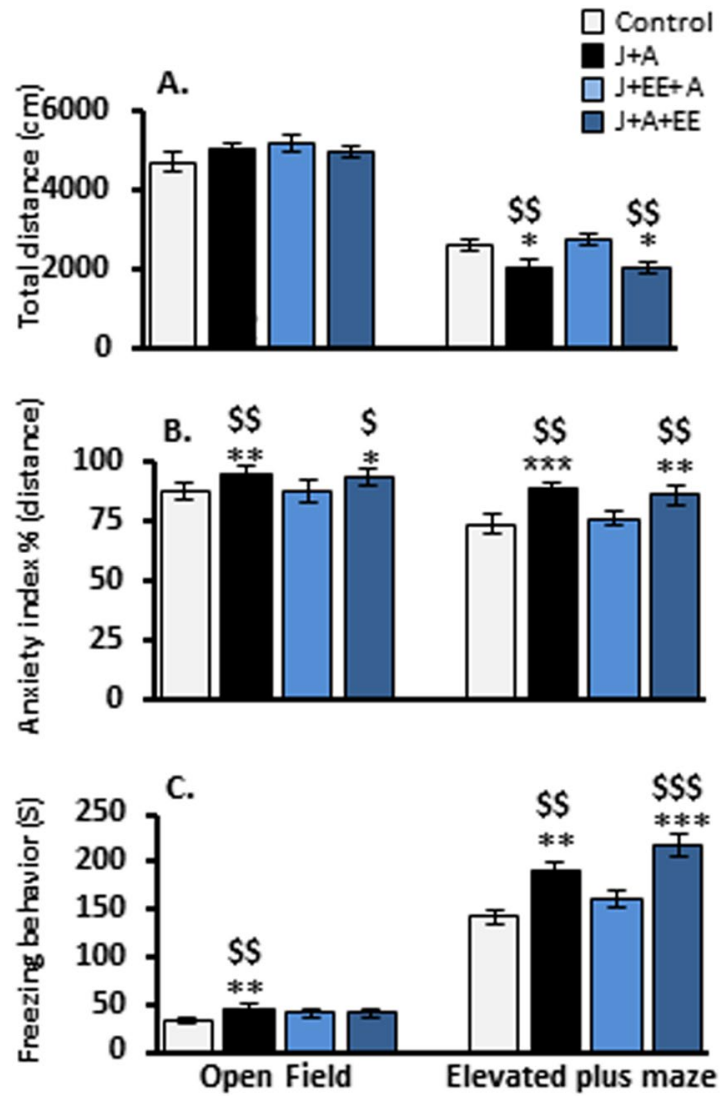

Figure 2. Exposure to enriched environment ameliorates the effects of stress if provided in juvenility, but not in adulthood. Group effects in the open field (OF) and elevated plus maze (EPM) paradigms. (A) Total distance. In the EPM, both $\mathrm{J}+\mathrm{A}$ and $\mathrm{J}+\mathrm{A}+\mathrm{EE}$ rats were less active compared to control and $\mathrm{J}+\mathrm{EE}+\mathrm{A}$ rats. (B) Anxiety index. In the $\mathrm{OF}$ and in the EPM, both $\mathrm{J}+\mathrm{A}$ and $\mathrm{J}+\mathrm{A}+\mathrm{EE}$ rats exhibited higher anxiety index compared to control and $\mathrm{J}+\mathrm{EE}+\mathrm{A}$ rats. (C) Freezing behavior. In the $\mathrm{OF}, \mathrm{J}+\mathrm{A}$ rats spent more time freezing compared to control and $\mathrm{J}+\mathrm{EE}+\mathrm{A}$ rats. In the $\mathrm{EPM}$, both $\mathrm{J}+\mathrm{A}$ and $\mathrm{J}+\mathrm{A}+\mathrm{EE}$ rats spent more time freezing compared to control and $\mathrm{J}+\mathrm{EE}+\mathrm{A}$ rats. All values are mean \pm SEM. Significant difference compared to control: *p $<0.05$, $* * \mathrm{p}<0.01, * * * \mathrm{p}<0.001$. Significant difference from $\mathrm{J}+\mathrm{EE}+\mathrm{A}:{ }^{\$} \mathrm{p}<0.05,{ }^{\$ \$} \mathrm{p}<0.01,{ }^{\$ \$} \mathrm{p}<0.001$.

Behavioral profiling. In the current study, we adopted the behavioral profiling approach as previously described by Ardi and colleagues ${ }^{18}$. In order to build individual behavioral profiles based on performance in the OF and EPM tests, we first explored the distribution of different behavioral values in the control group while referring to the performance of the control group as representative of the normal population. We used standard deviations in order to determine cut-off values for each behavioral parameter examined (Fig. 2A). Later, we compared the performance of each animal in the experiment to the relevant distribution curve of the control group. Rats that exhibited values that were outside the range between the lower and upper cut-off values in at least four out of the six behavioral measurements were classified as "affected" (Fig. 2B). 
Western blot. Brain harvesting, tissue collection, and western blot analysis were performed as previously described ${ }^{18}$. Immediately after the last behavioral assessment (PND 94), brains were collected and snap frozen for biochemical analysis ${ }^{16}$. Briefly, frozen tissue punches (diameter: $1 \mathrm{~mm}$; depth $1.5 \mathrm{~mm}$ ) were collected bilaterally from the dorsal dentate gyrus (dDG) and the dorsal Cornu Ammonis 1 (dCA1; both starting $-2.8 \mathrm{~mm}$ from Bregma, coronal) as well as the ventral DG and CA1 (vDG and vCA1; starting $-7.6 \mathrm{~mm}$ from Bregma, horizontal orientation) according to the Paxinos and Watson brain atlas ${ }^{19}$.

Tissue samples were homogenized in $300 \mu \mathrm{l}$ urea lysis buffer [ $1 \mathrm{mM}$ EDTA, $0.5 \%$ Triton X, $6 \mathrm{M}$ Urea, $100 \mu \mathrm{M}$ PMSF; Sigma-Aldrich, St. Louis, MO] containing protease and phosphatase inhibitors (Complete Ultra and PhosStop tablets [Roche Diagnostics, Mannheim, Germany]). Samples containing $10 \mu \mathrm{g}$ protein were loaded on a $12 \%$ SDS-polyacrylamide gel for electrophoresis, followed by semi-dry transfer to nitrocellulose membranes and blocking. Membranes were first incubated with primary antibodies (rabbit $\alpha \mathrm{GABA}_{\mathrm{A}} \alpha 1$ 1:2,500, Synaptic Systems, Göttingen, Germany; rabbit $\alpha$ GAPDH 1:2,000, Cell Signaling, Beverly, MA, USA; overnight at $4{ }^{\circ} \mathrm{C}$ ), then with a complementary secondary antibody ( $\alpha$ rabbit, polyclonal, $\left.1: 15,000\right)$. ECL Plus substrate (Advansta, Menlo Park, CA, USA) enabled chemiluminescence detection, and density of signals was analyzed with the Quantity One 1-D Analysis software. Ratios between optical density of target protein and control protein GAPDH were calculated for each sample and normalized first to a reference brain sample that was loaded on each gel for standardization across gels, and then to the mean density of the control group for each target and area.

Statistical analysis. First, differences in behavior and protein expression levels between the different experimental groups were tested using two-way ANOVA, followed by Fisher's protected least significant difference (PLSD) post hoc test. Second, after using the behavioral profiling, we used Pearson's chi-squared test in order to calculate the distribution of affected vs. unaffected populations. Last, relying on the behavioral profiling classification to "unaffected" and "affected" sub-populations in each experimental group, we then re-analyzed the protein expression data using Multivariate analysis for testing the effects of group, behavioral profile, and their interaction for each brain target and region. Significant interactions were further analyzed using planned comparisons within the different experimental groups and profiles. Specifically, we used one-way ANOVA followed by Fisher's PLSD post hoc test in order to test for differences between experimental groups in each behavioral profile (unaffected/ affected animals), and two tailed t-tests in order to test for differences between affected and unaffected rats within each experimental group. In order to avoid type 1 errors, we used the sequential Bonferroni correction to correct for multiple comparisons ${ }^{20}$.

\section{Results}

Averaged group effects in the open field and the elevated plus maze following exposure to stress and enriched environment. In order to evaluate the anxiolytic effects of exposure to juvenile and adult stress and exposure to an enriched environment (EE) during juvenility or in adulthood, we first compared the average group effects in the open field (OF) and elevated plus maze (EPM) paradigms, measuring total distance, anxiety index, and freezing behavior. No effects were found in the OF in the total distance travelled (twoway ANOVA; J + A: $\mathrm{F}(3,67)=1.22$, n.s.; $\mathrm{EE}: \mathrm{F}(3,67)=0.288$, n.s.). In the $\mathrm{EPM}$, two-way ANOVA revealed a significant main effect for both $\mathrm{J}+\mathrm{A}$ and $\mathrm{EE}$ exposure and the interaction between the two factors $(\mathrm{F}(3,67)=4.94$, $\mathrm{p}=0.03 ; \mathrm{F}(3,67)=5.24, \mathrm{p}=0.008 ; \mathrm{F}(3,67)=4.41, \mathrm{p}=0.007$, respectively). Further post hoc comparisons revealed a reduction in total activity in the EPM for both $\mathrm{J}+\mathrm{A}$ and $\mathrm{J}+\mathrm{A}+\mathrm{EE}$ groups compared to control $(\mathrm{p}=0.03$, $\mathrm{p}=0.021$ respectively) and to $\mathrm{J}+\mathrm{EE}+\mathrm{A}$ rats $(\mathrm{p}=0.008, \mathrm{p}=0.005$, respectively; Fig. $2 \mathrm{~A})$.

Calculating the anxiety index (distance in safe zone/[distance in safe zone + distance in higher risk zone]), two-way ANOVA revealed significant main effects both in the $\mathrm{OF}(\mathrm{J}+\mathrm{A}: \mathrm{F}(3,67)=8.71, \mathrm{p}=0.004$; EE: $\mathrm{F}(3,67)=4.84, \mathrm{p}=0.011$; Interaction: $\mathrm{F}(3,67)=5.027, \mathrm{p}=0.003)$ and in the $\mathrm{EPM}(\mathrm{J}+\mathrm{A}: \mathrm{F}(3,67)=15.94$, $\mathrm{p}=0.000$; $\mathrm{EE}: \mathrm{F}(3,67)=5.95, \mathrm{p}=0.004$; Interaction: $\mathrm{F}(3,67)=7.771, \mathrm{p}=0.000)$. Further post hoc comparisons revealed that in the $\mathrm{OF}$ both $\mathrm{J}+\mathrm{A}$ and $\mathrm{J}+\mathrm{A}+\mathrm{EE}$ rats showed elevated anxiety levels compared to control $(\mathrm{p}=0.004, \mathrm{p}=0.011$, respectively) and $\mathrm{J}+\mathrm{EE}+\mathrm{A}$ rats $(\mathrm{p}=0.006, \mathrm{p}=0.014$, respectively). In the EPM, post hoc comparisons revealed that both $\mathrm{J}+\mathrm{A}$ and $\mathrm{J}+\mathrm{A}+\mathrm{EE}$ rats showed elevated anxiety levels compared to control $(\mathrm{p}=0.000, \mathrm{p}=0.002$, respectively) and to $\mathrm{J}+\mathrm{EE}+\mathrm{A}$ rats $(\mathrm{p}=0.002, \mathrm{p}=0.01$, respectively; Fig. $2 \mathrm{~B})$.

Measuring freezing behavior as expressed by total freezing time, two-way ANOVA revealed significant main effects both in the $\mathrm{OF}(\mathrm{J}+\mathrm{A}: \mathrm{F}(3,67)=7.795, \mathrm{p}=0.007$; $\mathrm{EE}: \mathrm{F}(3,67)=4.281, \mathrm{p}=0.018$; Interaction: $\mathrm{F}(3,67)=3.932, \mathrm{p}=0.012)$ and the $\operatorname{EPM}(\mathrm{J}+\mathrm{A}: \mathrm{F}(3,67)=10.942, \mathrm{p}=0.002 ; \mathrm{EE}: \mathrm{F}(3,67)=10.224, \mathrm{p}=0.000$; Interaction: $\mathrm{F}(3,67)=11.652, \mathrm{p}=0.000)$. Further post hoc comparisons revealed that, in the $\mathrm{OF}, \mathrm{J}+\mathrm{A}$ rats showed increased freezing levels compared to control and $\mathrm{J}+\mathrm{EE}+\mathrm{A}$ rats $(\mathrm{p}=0.007, \mathrm{p}=0.005$, respectively). In the EPM, increased freezing levels were found for both $\mathrm{J}+\mathrm{A}$ and $\mathrm{J}+\mathrm{A}+\mathrm{EE}$ groups compared to control $(\mathrm{p}=0.002, \mathrm{p}=0.000$, respectively $)$ and $\mathrm{J}+\mathrm{EE}+\mathrm{A}$ rats $(\mathrm{p}=0.009, \mathrm{p}=0.000$, respectively; Fig. $2 \mathrm{C})$.

Assessing the prevalence of affected animals in the different groups by using the behavioral profiling approach. Behavioral profiling analysis, which takes into consideration individual differences in responses to stress and trauma, enables the classification of affected and unaffected individuals in a trauma-exposed population ${ }^{18,21}$ (Fig. 3A). Following the characterization of individual animals as "affected" or "unaffected", we re-analyzed the behavioral data presented in Fig. 2.

To determine the distribution of affected animals within the different groups, the number of individuals that exhibited values below the lower cut-off value or above the upper cut-off value in at least four out of the six behavioral parameters was compared between the different stress-exposure groups. Pearson $\chi^{2}$ analysis revealed a significant difference in the distribution of affected and unaffected individuals $\left(\chi^{2}\right.$ likelihood ratio $(3)=9.13$, $\mathrm{p}=0.021$; Fig. 3B). 
A.

a.

b.

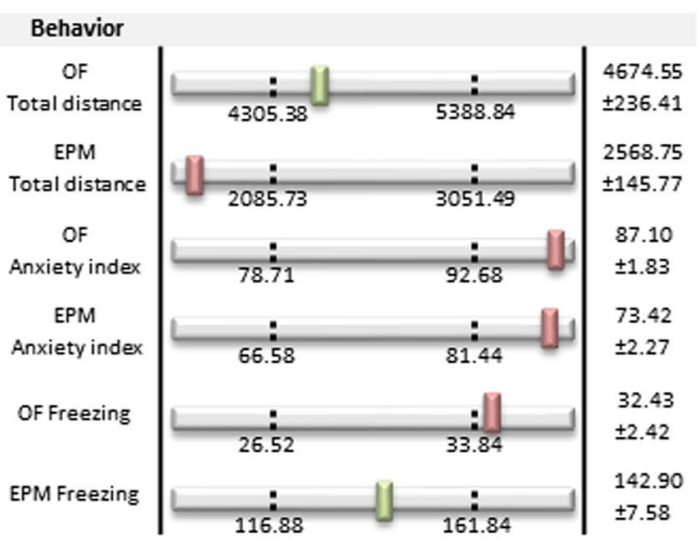

B.

\section{Unaffected \\ Affected}
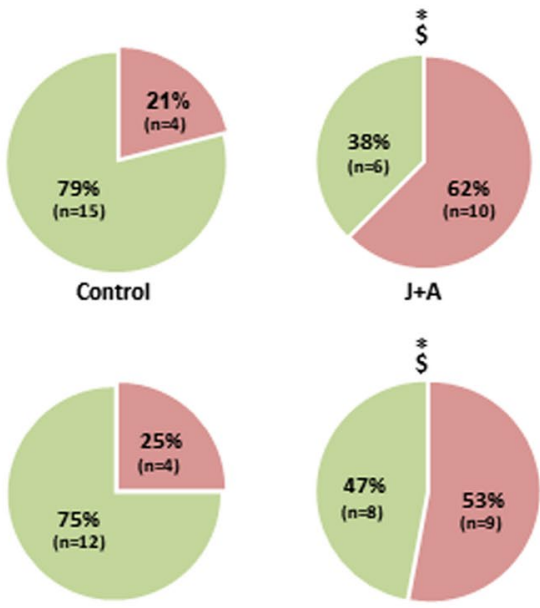

$J+E E+A$

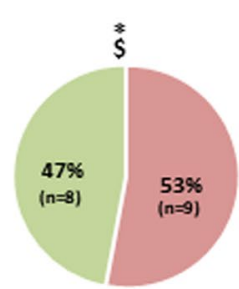

$J+A+E E$

Figure 3. Assessing the prevalence of affected animals in the different groups by using the behavioral profiling approach. (A) Schematic representation of the behavioral profiling approach. (a) Representative distribution curve of a given behavior in the control group. Standard deviations were used for calculating cut-off values based on the relevant distribution curve of the control group. (b) Representative example of a behavioral profile of an affected animal. In order to be classified as affected, an animal must exhibit values outside the range of the cut-off values in at least four out of the six behavioral parameters. (B) Prevalence of affected animals in the different groups By using the behavioral profiling approach, a significant higher proportion of affected animals was detected among both $\mathrm{J}+\mathrm{A}$ and $\mathrm{J}+\mathrm{A}+\mathrm{EE}$ rats compared to the proportion of affected animals among control and $\mathrm{J}+\mathrm{EE}+\mathrm{A}$ rats. All values are mean \pm SEM. Significant difference from control: $* \mathrm{p}<0.05$. Significant difference from $\mathrm{J}+\mathrm{EE}+\mathrm{A}:{ }^{\$} \mathrm{p}<0.05$.

Specific comparisons between groups using Pearson $\chi^{2}$ analysis revealed an increased prevalence of affected rats in the $\mathrm{J}+\mathrm{A}$ group compared to control and $\mathrm{J}+\mathrm{EE}+\mathrm{A}$ groups $\left(\chi^{2}\right.$ likelihood ratio $(1)=6.2, \mathrm{p}=0.013 ; \chi^{2}$ likelihood ratio( $(1)=4.69, \mathrm{p}=0.033$, respectively). A similar pattern of different distribution rates of affected animals was also found in $\mathrm{J}+\mathrm{A}+\mathrm{EE}$ rats compared to control and $\mathrm{J}+\mathrm{EE}+\mathrm{A}$ populations $\left(\chi^{2}\right.$ likelihood ratio $(1)=4.02$, $\mathrm{p}=0.047 ; \chi^{2}$ likelihood $\operatorname{ratio}(1)=3.94, \mathrm{p}=0.05$ respectively). There was no significant difference between the $\mathrm{J}+\mathrm{A}$ and $\mathrm{J}+\mathrm{A}+\mathrm{EE}$ groups.

Expression levels of $\mathrm{GABA}_{\mathrm{A}}$ receptor $\alpha 1$ subunit in dorsal and ventral hippocampal subregions following stress exposure and enriched environment intervention. Following previous findings, indicating increased expression levels of the $\mathrm{GABA}_{\mathrm{A}}$ receptor $\alpha 1$ subunit in the ventral hippocampus of stress-exposed animals ${ }^{16}$, we examined the effects of EE on the expression levels of $\mathrm{GABA}_{\mathrm{A}} \alpha 1$ in the dorsal and ventral hippocampus in the groups exposed to stress. In the dorsal hippocampus (Fig. 4A), two-way ANOVA revealed a significant effect for both $\mathrm{J}+\mathrm{A}$ and $\mathrm{EE}$ exposure and their interaction on $\mathrm{GABA}_{\mathrm{A}} \alpha 1$ expression levels in the dDG $(J+A: F(3,67)=21.24, p=0.000$; $E E: F(3,67)=3.57, p=0.034$; Interaction: $F(3,67)=7.556$, $\mathrm{p}=0.000)$. In the dCA1, two-way ANOVA revealed a significant effect for the $\mathrm{J}+\mathrm{A}$ exposure $(\mathrm{F}(3,67)=5.193$, $\mathrm{p}=0.026)$ and a borderline significant effect for both EE exposure and the J $\mathrm{A}$ and EE interaction (EE: $\mathrm{F}(3,67)=2.56, \mathrm{p}=0.081$; Interaction: $\mathrm{F}(3,67)=2.462, \mathrm{p}=0.071)$. Further post hoc comparisons revealed that, in the $\mathrm{dDG}$, both $\mathrm{J}+\mathrm{A}, \mathrm{J}+\mathrm{EE}+\mathrm{A}$, and $\mathrm{J}+\mathrm{A}+\mathrm{EE}$ showed increased $\alpha 1$ expression levels as compared to control $(\mathrm{p}=0.000, \mathrm{P}=0.038, \mathrm{p}=0.003$, respectively). In addition, $\mathrm{J}+\mathrm{A}$ rats showed increased $\alpha 1$ expression levels as compared to $\mathrm{J}+\mathrm{EE}+\mathrm{A}$ rats $(\mathrm{p}=0.018)$. In the $\mathrm{dCA} 1, \alpha 1$ expression levels in $\mathrm{J}+\mathrm{A}$ rats were significantly increased only compared to controls $(\mathrm{p}=0.013)$.

In the ventral hippocampus (Fig. 4B), two-way ANOVA revealed a significant effect for both $\mathrm{J}+\mathrm{A}$ and $\mathrm{EE}$ exposure and their interaction on $\mathrm{GABA}_{\mathrm{A}} \alpha 1$ expression levels in the $\mathrm{DG}(\mathrm{J}+\mathrm{A}: \mathrm{F}(3,67)=6.42, \mathrm{p}=0.013$; $\mathrm{EE}: \mathrm{F}(3,67)=7.201, \mathrm{p}=0.002$; Interaction: $\mathrm{F}(3,67)=7.697, \mathrm{p}=0.000)$ and the $\mathrm{CA} 1(\mathrm{~J}+\mathrm{A}: \mathrm{F}(3,67)=13.267$, $\mathrm{p}=0.001$; $\mathrm{EE}: \mathrm{F}(3,67)=8.699, \mathrm{p}=0.001$; Interaction: $\mathrm{F}(3,67)=6.855, \mathrm{p}=0.000)$. Further post hoc comparisons revealed increased $v D G \alpha 1$ expression levels in both $\mathrm{J}+\mathrm{A}$ and $\mathrm{J}+\mathrm{A}+\mathrm{EE}$ rats compared to control $(\mathrm{p}=0.007$, $p=0.000$, respectively) and $J+E E+A$ rats $(p=0.017, p=0.000$, respectively). In the vCA 1 , increased $\alpha 1$ expression levels were found in $\mathrm{J}+\mathrm{A}$ rats compared to control $(\mathrm{p}=0.001), \mathrm{J}+\mathrm{EE}+\mathrm{A}(\mathrm{p}=0.000)$, and to $\mathrm{J}+\mathrm{A}+\mathrm{EE}$ $(\mathrm{p}=0.032)$. Additionally, a significant increase in $\alpha 1$ expression levels was found in $\mathrm{J}+\mathrm{A}+\mathrm{EE}$ rats compared to $\mathrm{J}+\mathrm{EE}+\mathrm{A}$ rats $(\mathrm{p}=0.043)$. 
A.

Dorsal Hippocampus
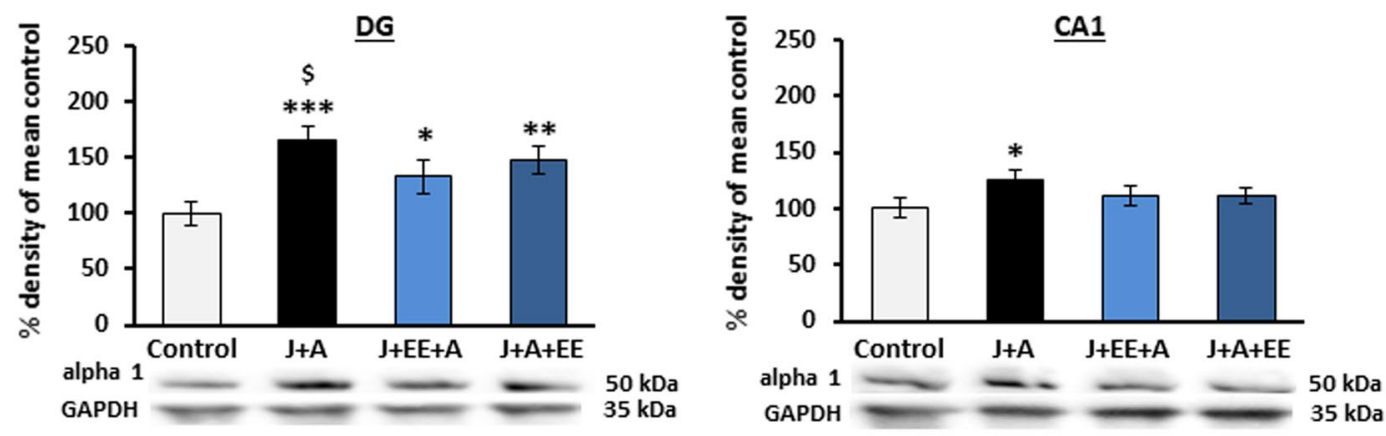

B.

Ventral Hippocampus
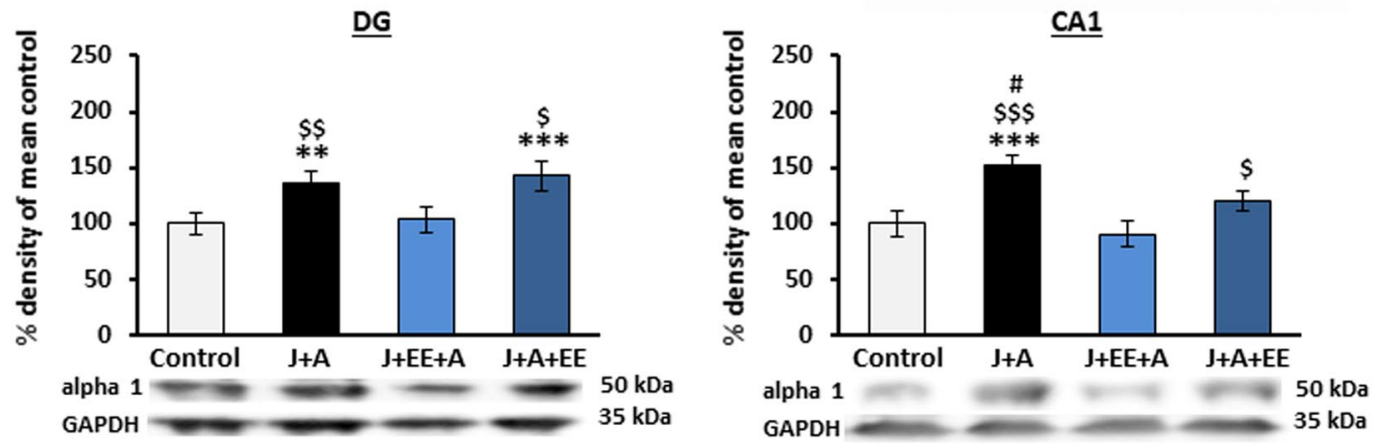

Figure 4. Alterations in expression levels of $\mathrm{GABA}_{\mathrm{A}}$ receptor $\alpha 1$ subunit in dorsal and ventral hippocampal subregions following stress exposure and environmental enrichment. (A) Dorsal hippocampus. In the dDG, expression levels of $\mathrm{GABA}_{\mathrm{A}} \alpha 1$ were increased in all groups compared to control rats. In the $\mathrm{dCA} 1, \alpha 1$ expression levels were increased only in J + A rats compared to control. (B) Ventral hippocampus. In the vDG, both $\mathrm{J}+\mathrm{A}$ and $\mathrm{J}+\mathrm{A}+\mathrm{EE}$ rats showed higher expression levels of $\mathrm{GABA}_{\mathrm{A}}$ receptor $\alpha 1$ compared to control and $\mathrm{J}+\mathrm{EE}+\mathrm{A}$ rats. In the $\mathrm{vCA} 1$, while $\mathrm{J}+\mathrm{A}$ rats showed higher $\alpha 1$ expression levels compared to all other groups, $\mathrm{J}+\mathrm{A}+\mathrm{EE}$ rats showed increased $\alpha 1$ expression compared to $\mathrm{J}+\mathrm{EE}+\mathrm{A}$ rats. All values are $\%$ density of mean control (mean \pm SEM). Significant difference from control: $* p<0.05, * * p<0.01, * * * p<0.001$. Significant difference from J $+E E+A:{ }^{\$} \mathrm{p}<0.05,{ }^{\$ \$} \mathrm{p}<0.01,{ }^{\$ \$} \mathrm{p}<0.001$. Significant difference from $\mathrm{J}+\mathrm{A}+\mathrm{EE}: \# \mathrm{p}<0.05$. Sample blots are line blots from the same gel.

Assessing expression levels of $\mathrm{GABA}_{\mathrm{A}}$ receptor $\alpha 1$ subunit in dorsal and ventral hippocampal subregions following the behavioral profiling characterization. Analysis of the effects of EE intervention on $\alpha 1$ expression levels using group averages might mask expression alterations associated with the different behavioral profiles of individuals, as previously reported ${ }^{16}$. We thus re-analyzed the levels of expression according to the categorization of affected vs. unaffected individuals. First, Multivariate analysis for $\mathrm{GABA}_{\mathrm{A}}$ receptor $\alpha 1$ expression levels in each subregion was applied, assessing the interactions between exposure and behavioral profiles (unaffected vs. affected animals). In the dorsal hippocampus, two-way ANOVA did not reveal any significant interactions in either the $\mathrm{dDG}$ or the dCA1 (Fig. $5 \mathrm{~A}: \mathrm{F}(3,60)=0.51$, n.s. and $\mathrm{F}(3,60)=1.07$, n.s., respectively). By contrast, in the ventral hippocampus, two-way ANOVA revealed significant interactions in both the $v D G$ and vCA1 (Fig. $5 \mathrm{~B}: \mathrm{F}(3,60)=2.91, \mathrm{p}=0.041$ and $\mathrm{F}(3,60)=3.33 \mathrm{p}=0.025$, respectively).

Second, comparisons using one-way ANOVA revealed a significant effect for Group among unaffected rats in both the vDG and vCA1 (ANOVA between groups of unaffected populations $\mathrm{F}(3,40)=15.43, \mathrm{p}=0.000$ and $\mathrm{F}(3,39)=11.64, \mathrm{p}=0.000$, respectively). In the $\mathrm{vDG}$, further post hoc comparisons revealed a specific increase in $\mathrm{GABA}_{\mathrm{A}}$ receptor $\alpha 1$ expression in unaffected rats from both $\mathrm{J}+\mathrm{A}$ and $\mathrm{J}+\mathrm{A}+\mathrm{EE}$ groups compared to unaffected rats from the control $(p=0.000 . p=0.000$, respectively $)$ and $J+E E+A$ groups $(p=0.001, p=0.000$, respectively). In the vCA1, further post hoc comparisons revealed a specific increase in $\mathrm{GABA}_{\mathrm{A}}$ receptor $\alpha 1$ expression levels in unaffected rats from both $\mathrm{J}+\mathrm{A}$ and $\mathrm{J}+\mathrm{A}+\mathrm{EE}$ groups compared to unaffected rats from the control group $(p=0.000 . p=0.007$, respectively) and from $J+E E+A$ rats $(p=0.000, p=0.002$, respectively). This was further confirmed by a direct comparison of VDG and vCA1 $\alpha 1$ levels in unaffected vs. affected J $+\mathrm{A}$ rats 
A.
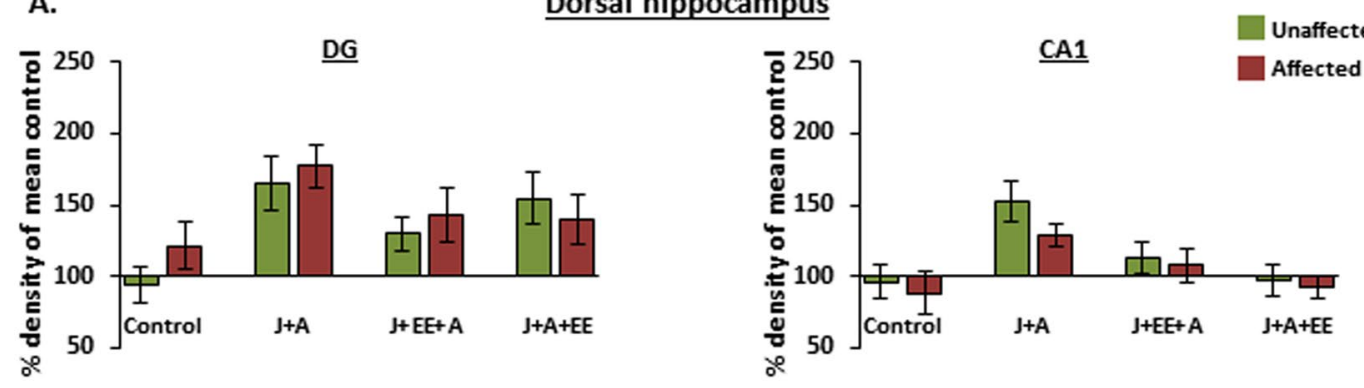

B.

Ventral hippocampus
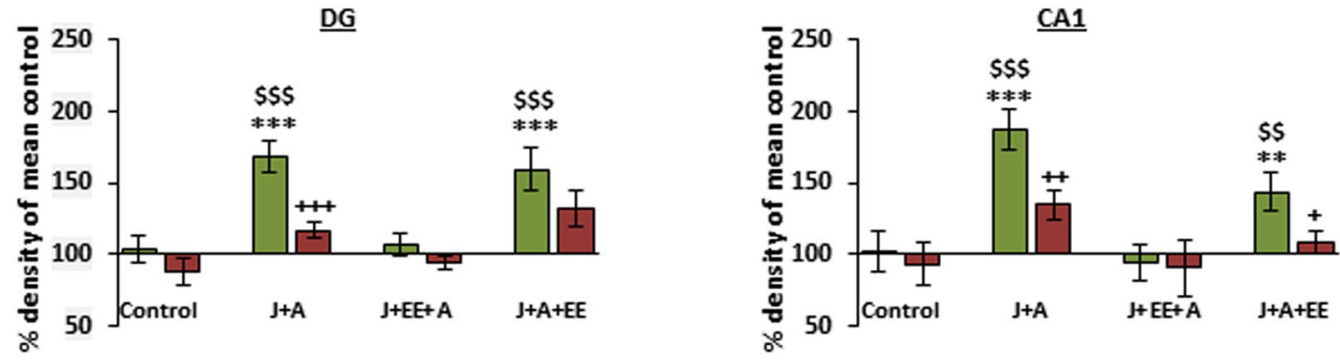

Figure 5. Increased expression levels of $\mathrm{GABA}_{\mathrm{A}}$ receptor $\alpha 1$ subunit in the ventral hippocampal subregions of stress resilient animals. (A) Dorsal hippocampus. No significant interactions of stress-exposure group $\mathrm{x}$ behavioral profile were observed in either the dDG or dCA1. (B) Ventral hippocampus. For GABA $A_{A}$ receptor $\alpha 1$ in the vDG, a significant interaction of stress-exposure group $\mathrm{x}$ behavioral profile was observed. An increased expression of $\mathrm{GABA}_{\mathrm{A}}$ receptor $\alpha 1$ was observed in unaffected rats of both $\mathrm{J}+\mathrm{A}$ and $\mathrm{J}+\mathrm{A}+\mathrm{EE}$ groups, compared to unaffected rats from the control and $\mathrm{J}+\mathrm{EE}+\mathrm{A}$ groups. Direct comparison also revealed an increase in $\mathrm{GABA}_{\mathrm{A}}$ receptor $\alpha 1$ levels in unaffected rats compared to affected rats within the $\mathrm{J}+\mathrm{A}$ group. A similar expression pattern was observed for $\mathrm{GABA}_{\mathrm{A}}$ receptor $\alpha 1$ in the vCA1 of unaffected rats in both $\mathrm{J}+\mathrm{A}$ and $\mathrm{J}+\mathrm{A}+\mathrm{EE}$ groups, compared to unaffected rats from the control and $\mathrm{J}+\mathrm{EE}+\mathrm{A}$ groups. Here, direct comparison revealed an increase in $\mathrm{GABA}_{\mathrm{A}}$ receptor $\alpha 1$ levels in unaffected rats compared to affected rats in both $\mathrm{J}+\mathrm{A}$ and the $\mathrm{J}+\mathrm{A}+\mathrm{EE}$ groups. All values are $\%$ density of mean control (mean $\pm \mathrm{SEM})$. Significantly different from unaffected control: $* * \mathrm{p}<0.01, * * * \mathrm{p}<0.001$. Significant difference from unaffected $\mathrm{J}+\mathrm{EE}+\mathrm{A}$ : ${ }^{\$} \mathrm{p}<0.01,{ }^{\$ \$} \mathrm{p}<0.001$. Significant difference unaffected vs. affected rats: ${ }^{+} \mathrm{p}<0.05,{ }^{++} \mathrm{p}<0.01,{ }^{+++} \mathrm{p}<0.001$.

$(\mathrm{T}(14)=4.483, \mathrm{p}=0.000$ and $\mathrm{T}(13)=6.821 \mathrm{p}=0.012$, respectively) and vCA $1 \alpha 1$ levels in unaffected vs. affected $\mathrm{J}+\mathrm{A}+\mathrm{EE}$ rats $(\mathrm{T}(15)=4.94, \mathrm{p}=0.026)$. No expression regulation was observed in affected animals (ANOVA between groups of affected populations $\mathrm{F}(3,26)=2.14$, n.s. and $\mathrm{F}(3,26)=2.69$, n.s., respectively).

\section{Discussion}

Our results corroborate the findings that increased expression levels of the $\mathrm{GABA}_{\mathrm{A}}$ receptor $\alpha 1$ subunit in the ventral hippocampus is associated with stress resilience ${ }^{18}$, using a different experimental context. Furthermore, our findings demonstrate the potential importance of early interventions, from pre-puberty and into adulthood, in building up stress resilience and in reducing the negative impact of juvenile stress on coping with trauma in adulthood. Juvenility is a stress-sensitive period during which exposure to aversive experiences increases the probability of developing psychopathologies in the face of trauma later in life $e^{6-10,17,18}$. However, juvenility may also be an effective period for initiating intervention. We have already demonstrated that selective serotonin reuptake inhibitors (SSRIs) ${ }^{11}$ or $\mathrm{EE}^{12}$ interventions can reduce the impact of juvenile aversive experiences into adulthood. Here we further demonstrate that EE later in life, from immediately following the exposure to trauma in adulthood, is no longer effective (Fig. 6).

The current results further demonstrate that EE-associated increased resilience is mediated by a mechanism that is not dependent on increased $\alpha 1$ expression in the ventral hippocampus. Resilience-associated increased $\alpha 1$ expression in the ventral hippocampus was found in unaffected individuals of the $\mathrm{J}+\mathrm{A}$ and $\mathrm{J}+\mathrm{A}+\mathrm{EE}$ groups, but not in unaffected individuals of the $\mathrm{J}+\mathrm{EE}+\mathrm{A}$ group (Fig. 6).

What may mediate the effects of EE? EE has been associated with enhanced neurogenesis and increased expression of brain derived neurotrophic factor (BDNF), and those are often suggested to mediate the beneficial effects of EE on learning and memory and on stress resilience ${ }^{22,23}$. Exposure to stress and trauma ${ }^{24}$, including exposure to pre-pubertal stress ${ }^{25}$, is suggested to result in reduced expression of BDNF and impaired neurogenesis in the hippocampus, and EE is assumed to reverse these effects by enhancing hippocampal neurogenesis and BDNF expression ${ }^{22,26-32}$. In addition, EE was found to prevent the reduction in hippocampal volume in an animal model of PTSD ${ }^{33}$. Reduced hippocampal volume has been suggested in human PTSD together with the possibility that it may result from the impaired neurogenesis associated with exposure to stress and trauma ${ }^{24}$. Similarly, chronic stress exposure, a well-established model of mood disorders, results in mood-related symptoms 

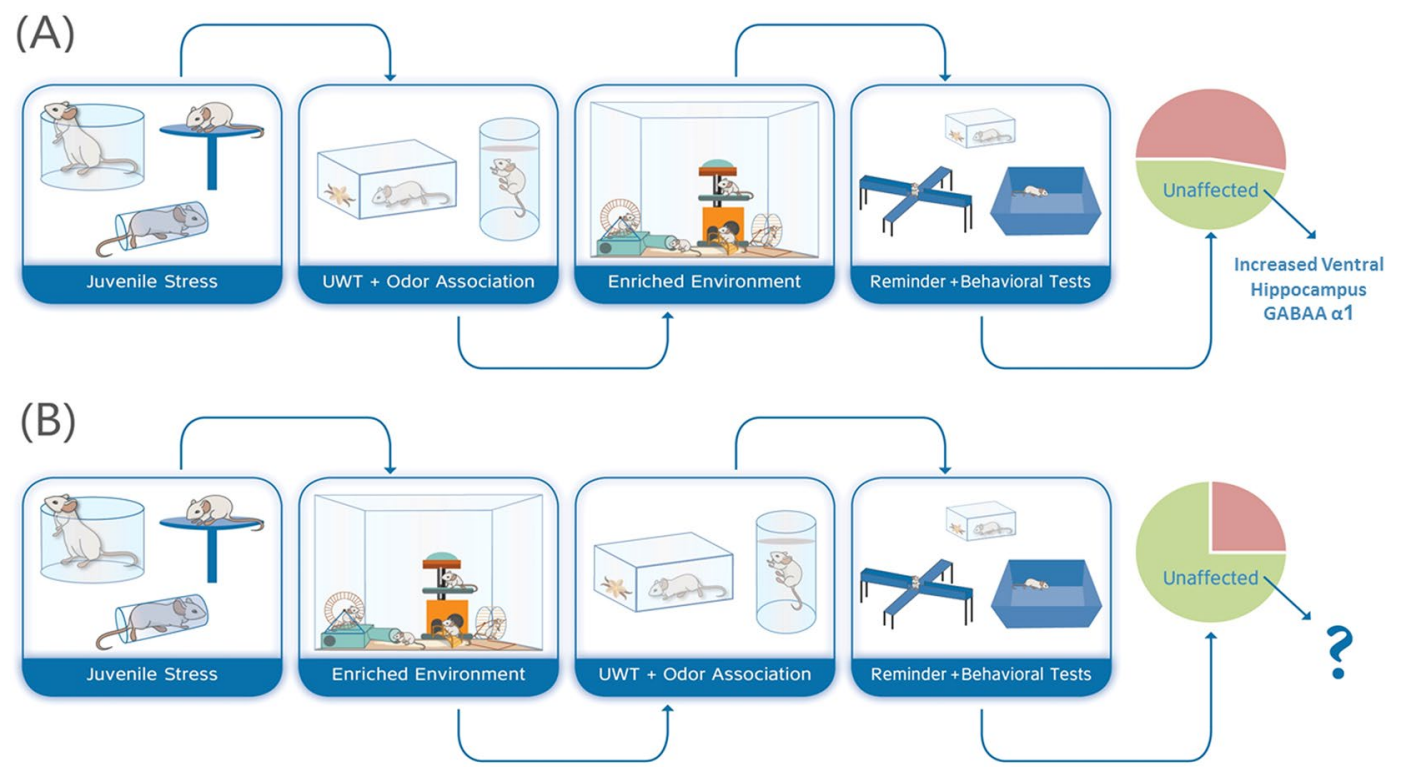

Figure 6. A graphic summary: EE during juvenility reduces the impact of juvenile stress, an effect that is not mediated by increased expression of $\mathrm{GABA}_{\mathrm{A}}$ receptor $\alpha 1$. (A) EE for one month following exposure to $\mathrm{J}+\mathrm{A}$ stress is not effective in reducing the adverse impact of the stress exposure. The proportion of affected animals is similar to the proportion of affected animals following $J+A$ stress without exposure to EE. A significant increase in the expression of $\alpha 1$ in the ventral hippocampus was found in animals that were exposed to the $\mathrm{J}+$ A protocol and did not develop PTSD-like symptoms, as previously reported for unaffected animals without exposure to $\mathrm{EE}^{18}$. This result supports the notion that expression of $\alpha 1$ in the ventral hippocampus is associated with increased stress resilience. (B) EE for one month following exposure to juvenile stress was effective in reducing the negative impact of juvenile stress on coping with trauma in adulthood. However, resilience following the exposure to EE was not associated with increased expression of $\alpha 1$ in the ventral hippocampus, suggesting that the neural mechanisms involved in EE-associated resilience are different from those associated with naturally-occurring stress resilience.

reminiscent of depressive symptoms in mood disorders ${ }^{34}$. In addition, antidepressants such as SSRIs were found to have effects similar to EE, including increased hippocampal neurogenesis and BDNF expression ${ }^{34}$. Together, these findings have led to the conception that upregulation of BDNF and subsequent induction of neurogenesis mediate the effects induced by both SSRIs and EE in stress-related and mood disorders ${ }^{34,35}$, and that hippocampal neurogenesis plays a major role in stress resilience ${ }^{23}$.

There are, however, opposing observations, which indicate that hippocampal neurogenesis is not required for at least some behavioral effects of enriched environment ${ }^{36,37}$ or for some effects of anti-depressants ${ }^{38,39}$. Furthermore, resilience is found also without exposure to EE or to SSRIs ${ }^{18}$. These findings point to the involvement of additional factors.

Such proposed additional factors are alterations in the activity of GABAergic interneurons, which are important modulators of neural activity and plasticity. Alterations in GABAergic neurotransmission have been implicated in stress, trauma, and PTSD ${ }^{14-17}$. PTSD has been found to be associated with reduced GABA $_{\mathrm{A}}$ benzodiazepine receptor binding ${ }^{40}$, and although the use of benzodiazepines as medication in PTSD is under debate, these drugs are nevertheless widely prescribed to PTSD patients ${ }^{41}$. The effects of EE on neurogenesis have been found to involve effects on GABAergic interneurons ${ }^{42,43}$. Furthermore, BDNF, a key mediator of protective EE effects after stress ${ }^{22,23}$, has been found to be involved in modulating GABAergic neurotransmission in relation to both stress vulnerability and stress resilience ${ }^{44,45}$. Stress-induced alterations in neurosteroid biosynthesis and changes in $\mathrm{GABA}_{\mathrm{A}}$ receptor subunit expression have already been suggested as potential biomarkers and targets for the development of new PTSD treatments ${ }^{46}$. Interestingly, pre-pubertal stress has been found to alter the expression of both neurosteroids ${ }^{47}$ and $\mathrm{GABA}_{\mathrm{A}}$ receptor $\alpha$ subunits expression ${ }^{18,48,49}$. The current results, indicating that alteration of the expression of the $\mathrm{GABA}_{\mathrm{A}}$ receptor $\alpha 1$ subunit specifically in resilient individuals, further emphasize the contribution of altered GABAergic neurotransmission in trauma-related vulnerability and resilience ${ }^{44}$.

The resilience-associated alteration in $\alpha 1$ expression in our study was relatively selective to the ventral hippocampus. This result supports our previous findings describing a selective upregulation of the $\mathrm{GABA}_{\mathrm{A}}$ receptor $\alpha 1$ subunit in the ventral hippocampus of resilient animals ${ }^{18}$. The hippocampus is suggested to exert differential contributions to cognition and emotionality along its septo-temporal axis in humans, reflecting the hippocampal dorsal-ventral axis in rodents. For example, the dorsal hippocampus has been implicated in cognitive functions such as spatial learning and memory, while the ventral hippocampus has been associated with emotional responses and in particular with responses to stress ${ }^{50}$. Indeed, stress-induced alterations are frequently more pronounced in the ventral hippocampus ${ }^{51}$. 
The ventral hippocampus modulates the hypothalamo-pituitary-adrenocortical axis response ${ }^{52,53}$, presumably via the ventral subiculum ${ }^{3,54}$. Exposure to chronic stress affects neurogenesis predominantly in the ventral hippocampus ${ }^{55}$. It has been suggested that the effects of antidepressants on BDNF, neurogenesis, and behavior are mediated mainly by their effects on the ventral hippocampus ${ }^{34}$. More specifically, it is suggested that preferential regulation of neurogenesis in the ventral hippocampus contributes to stress resilience and antidepressant action $^{23,56,57}$. Thus, a selective upregulation of the $\mathrm{GABA}_{\mathrm{A}}$ receptor $\alpha 1$ subunit in stress resilient animals may comprise an additional mechanism of emotion-related changes of the ventral hippocampus. These resilience-related mechanisms can presumably be achieved more effectively during juvenility, adolescence, and into young adulthood, as previously suggested ${ }^{11,12}$.

Examining individual differences with regards to stress vulnerability and resilience appears to be critical for our understanding of the neural basis of stress-related psychopathology and stress resilience. The current results, together with previous findings $s^{17,18,23,58}$, emphasize that exposure to trauma, which may lead to the development of stress-related psychopathologies, such as PTSD, involves the activation of numerous neuronal responses and alterations. Some of these alterations presumably underlie the development of pathology. However, some of these changes rather reflect attempts of the system to regain control and support stress-resilience ${ }^{17}$. Dissociating between vulnerability/pathology-associated alterations and those associated with stress resilience is clearly imperative for successful translation of preclinical findings into effective treatment. Introducing behavioral profiling analysis and distinguishing between exposed-affected and exposed-unaffected individuals is an important tool in that respect, which here led to the more accurate association of the elevated expression of the $\mathrm{GABA}_{\mathrm{A}}$ receptor subunit $\alpha 1$ with stress resilience. Assessing the impact of EE manipulation on the proportion of affected versus unaffected individuals rather than on the averaged group results helped demonstrate the relative effectiveness of pre-puberty EE intervention compared to the impact of a similar manipulation following exposure to trauma in adulthood. Exposure to aversive experiences in childhood is a known risk factor for developing psychopathologies later in life ${ }^{1-3}$. The current findings emphasize the importance of considering early interventions, during pre-puberty, in order to reduce the likelihood of developing psychopathologies later in life.

\section{References}

1. Bremner, J. D., Southwick, S. M., Johnson, D. R., Yehuda, R. \& Charney, D. S. Childhood physical abuse and combat-related posttraumatic stress disorder in Vietnam veterans. Am J Psychiatry 150(2), 235-239 (1993).

2. Heim, C. \& Nemeroff, C. B. The role of childhood trauma in the neurobiology of mood and anxiety disorders: preclinical and clinical studies. Biol. Psychiatry 49(12), 1023-1039 (2001).

3. McLaughlin, K. A. et al. Childhood adversities and post-traumatic stress disorder: evidence for stress sensitisation in the World Mental Health Surveys. Br J Psychiatry 211(5), 280-288 (2017).

4. Spear, L. P. The adolescent brain and age related behavioral manifestations. Neurosci Behave Rev 24, 417-463 (2000).

5. Spear, L. P. Adolescent brain development and animal models. Ann NY Acad Sci 1021, 23-26 (2004).

6. Avital, A. \& Richter-Levin, G. Exposure to juvenile stress exacerbates the behavioural consequences of exposure to stress in the adult rat. Int. J. Neuropsychopharmacol 8(2), 163-173 (2005).

7. Toledo-Rodriguez, M. \& Sandi, C. Stress during Adolescence Increases Novelty Seeking and Risk-Taking Behavior in Male and Female Rats. Front Behav Neurosci. 5, 17 (2011).

8. Horovitz, O., Tsoory, M. M., Hall, J., Jacobson-Pick, S. \& Richter-Levin, G. Post-weaning to pre-pubertal ('juvenile’) stress: a model of induced predisposition to stress-related disorders. Neuroendocrinology 95(1), 56-64 (2012).

9. Brydges, N. M., Hall, L., Nicolson, R., Holmes, M. C. \& Hall, J. The effects of juvenile stress on anxiety, cognitive bias and decision making in adulthood: a rat model. PLoS One, 7(10), e48143 (2012).

10. Fuentes, S., Carrasco, J., Armario, A. \& Nadal, R. Behavioral and neuroendocrine consequences of juvenile stress combined with adult immobilization in male rats. Horm Behav 66(3), 475-486 (2014).

11. Ariel, L., Inbar, S., Edut, S. \& Richter-Levin, G. Fluoxetine treatment is effective in a rat model of childhood-induced post-traumatic stress disorder. Transl Psychiatry 7(11), 1260 (2017).

12. Ilin, Y. \& Richter-Levin, G. Enriched environment experience overcomes learning deficits and depressive-like behavior induced by juvenile stress. PLoS One, 4(1), e4329 (2009).

13. Shtoots, L., Richter-Levin, G., Hugeri, O. \& Anunu, R. Juvenile stress leads to long-term immunological metaplasticity-like effects on inflammatory responses in adulthood. Neurobiol Learn Mem, 154, 12-21 (2018).

14. Vaiva, G. et al. Relationship between posttrauma GABA plasma levels and PTSD at 1-year follow-up. Am. J. Psychiatry 163, 1446-1448 (2006).

15. Skilbeck, K. J., Johnston, G. A. \& Hinton, T. Stress and GABA receptors. J. Neurochem 112, 1115-1130 (2010).

16. Schür, R. R. et al. Development of psychopathology in deployed armed forces in relation to plasma GABA levels. Psychoneuroendocrinology 73, 263-270 (2016).

17. Albrecht, A. et al. Neurobiological consequences of juvenile stress: A GABAergic perspective on risk and resilience. Neurosci Biobehav Rev 74(Pt A), 21-43 (2017).

18. Ardi, Z., Albrecht, A., Richter-Levin, A., Saha, R. \& Richter-Levin, G. Behavioral profiling as a translational approach in an animal model of posttraumatic stress disorder. Neurobiol Dis 88, 139-47 (2016).

19. Paxinos, G. A. W. C., \& Watson, C. The rat brain atlas in stereotaxic coordinates (San Diego: Academic, 1998).

20. Holm, S. A simple sequentially rejective multiple test procedure. Scandinavian journal of statistics 6, 65-70 (1979).

21. Ritov, G., Boltyansky, B. \& Richter-Levin, G. A novel approach to PTSD modeling in rats reveals alternating patterns of limbic activity in different types of stress reaction. Mol Psychiatry 21(5), 630-641 (2016).

22. Bekinschtein, P., Oomen, C. A., Saksida, L. M. \& Bussey, T. J. Effects of environmental enrichment and voluntary exercise on neurogenesis, learning and memory, and pattern separation: BDNF as a critical variable? Semin Cell Dev Biol 22(5), 536-542 (2011).

23. Levone, B. R., Cryan, J. F. \& O'Leary, O. F. Role of adult hippocampal neurogenesis in stress resilience. Neurobiol Stress 1, 147-155 (2014).

24. Rubin de Celis, M. F. et al. The effects of stress on brain and adrenal stem cells. Mol Psychiatry 21(5), 590-593 (2016).

25. Bazak, N. et al. Pre-pubertal stress exposure affects adult behavioral response in association with changes in circulating corticosterone and brain-derived neurotrophic factor. Psychoneuroendocrinology 34(6), 844-858 (2009).

26. Rossi, C. et al. Brain-derived neurotrophic factor (BDNF) is required for the enhancement of hippocampal neurogenesis following environmental enrichment. Eur J Neurosci 24(7), 1850-1856 (2006).

27. Griffin, E. W., Bechara, R. G., Birch, A. M. \& Kelly, A. M. Exercise enhances hippocampal-dependent learning in the rat: evidence for a BDNF-related mechanism. Hippocampus 19, 973-980 (2009). 
28. Schloesser, R. J., Lehmann, M., Martinowich, K., Manji, H. K. \& Herkenham, M. Environmental enrichment requires adult neurogenesis to facilitate the recovery from psychosocial stress. Mol Psychiatry 15(12), 1152-1163 (2010).

29. Fang, Z. H. et al. Effect of treadmill exercise on the BDNF-mediated pathway in the hippocampus of stressed rats. Neurosci Res 76, 187-194 (2013)

30. Yu, J. L., Ma, L., Ma, L. \& Tao, Y. Z. Voluntary wheel running enhances cell proliferation and expression levels of BDNF, IGF1 and WNT4 in dentate gyrus of adult mice. Sheng Li Xue Bao 66(5), 559-568 (2014).

31. Zhang, X. Q. et al. Increased protein expression levels of pCREB, BDNF and SDF-1/CXCR4 in the hippocampus may be associated with enhanced neurogenesis induced by environmental enrichment. Mol Med Rep 14(3), 2231-2237 (2016).

32. Tanichi, M. et al. Differential effects of voluntary wheel running and toy rotation on the mRNA expression of neurotrophic factors and FKBP5 in a post-traumatic stress disorder rat model with the shuttle-box task. Biochem Biophys Res Commun 501(1), 307-312 (2018).

33. Golub, Y. et al. Reduced hippocampus volume in the mouse model of Posttraumatic Stress Disorder. J Psychiatr Res 45(5), 650-659 (2011).

34. Mahar, I., Bambico, F. R., Mechawar, N. \& Nobrega, J. N. Stress, serotonin, and hippocampal neurogenesis in relation to depression and antidepressant effects. Neurosci Biobehav Rev 38, 173-92 (2014).

35. Masi, G. \& Brovedani, P. The hippocampus, neurotrophic factors and depression: possible implications for the pharmacotherapy of depression. CNS Drugs 25(11), 913-931 (2011).

36. Meshi, D. et al. Hippocampal neurogenesis is not required for behavioral effects of environmental enrichment. Nat Neurosci 9 , 729-731 (2006).

37. Bessa, J. M. et al. The mood-improving actions of antidepressants do not depend on neurogenesis but are associated with neuronal remodeling. Mol Psychiatry 14(8), 764-773 (2009).

38. Holick, K. A., Lee, D. C., Hen, R. \& Dulawa, S. C. Behavioral effects of chronic fluoxetine in BALB/cJ mice do not require adult hippocampal neurogenesis or the serotonin 1A receptor. Neuropsychopharmacology 33(2), 406-417 (2008).

39. David, D. J. et al. Neurogenesis-dependent and -independent effects of fluoxetine in an animal model of anxiety/depression. Neuron 62(4), 479-493 (2009).

40. Geuze, E. Reduced GABAA benzodiazepine receptor binding in veterans with post-traumatic stress disorder. Mol Psychiatry 13(1), 74-83 (2008).

41. Loeffler, G., Coller, R., Tracy, L. \& Derderian, B. R. Prescribing Trends in US Active Duty Service Members with Posttraumatic Stress Disorder: A Population-Based Study from 2007-2013. J Clin Psychiatry, 7(4), 9 (2018).

42. Segovia, G., Yagüe, A. G., García-Verdugo, J. M. \& Mora, F. Environmental enrichment promotes neurogenesis and changes the extracellular concentrations of glutamate and GABA in the hippocampus of aged rats. Brain Res Bull 70(1), 8-14 (2006).

43. Sampedro-Piquero, P., Castilla-Ortega, E., Zancada-Menendez, C., Santín, L. J. \& Begega, A. Environmental enrichment as a therapeutic avenue for anxiety in aged Wistar rats: Effect on cat odor exposition and GABAergic interneurons. Neuroscience 330, $17-25$ (2016).

44. Kim, J. et al. Brain-derived neurotropic factor and GABAergic transmission in neurodegeneration and neuroregeneration. Neural Regen Res 12(10), 1733-1741 (2017)

45. Brady, M. L. et al. Depolarizing, inhibitory GABA type A receptor activity regulates GABAergic synapse plasticity via ERK and BDNF signaling. Neuropharmacology 128, 324-339 (2018).

46. Locci, A. \& Pinna, G. Neurosteroid biosynthesis down-regulation and changes in GABAA receptor subunit composition: a biomarker axis in stress-induced cognitive and emotional impairment. Br J Pharmacol 174(19), 3226-3241 (2017).

47. Avital, A., Ram, E., Maayan, R., Weizman, A. \& Richter-Levin, G. Effects of early-life stress on behavior and neurosteroid levels in the rat hypothalamus and entorhinal cortex. Brain Res Bull 68(6), 419-424 (2006).

48. Jacobson-Pick, S., Elkobi, A., Vander, S., Rosenblum, K. \& Richter-Levin, G. Juvenile stress-induced alteration of maturation of the GABAA receptor alpha subunit in the rat. Int J Neuropsychopharmacol 11(7), 891-903 (2008).

49. Jacobson-Pick, S. \& Richter-Levin, G. Short- and long-term effects of juvenile stressor exposure on the expression of GABAA receptor subunits in rats. Stress 15(4), 16-24 (2012).

50. Fanselow, M. S. \& Dong, H. W. Are the dorsal and ventral hippocampus functionally distinct structures? Neuron 65(1), 7-19 (2010).

51. Floriou-Servou, A. et al. Distinct Proteomic, Transcriptomic, and Epigenetic Stress Responses in Dorsal and Ventral Hippocampus. Biol Psychiatry 84(7), 531-541 (2018).

52. Casady, R. L. \& Taylor, A. N. Effect of electrical stimulation of the hippocampus upon corticosteroid levels in the freely-behaving, non-stressed rat. Neuroendocrinology 20(1), 68-78 (1976).

53. Jankord, R. \& Herman, J. P. Limbic regulation of hypothalamo-pituitary-adrenocortical function during acute and chronic stress. Ann N Y Acad Sci 1148, 64-73 (2008).

54. Herman, J. P., Cullinan, W. E., Morano, M. I., Akil, H. \& Watson, S. J. Contribution of the ventral subiculum to inhibitory regulation of the hypothalamo-pituitary-adrenocortical axis. J Neuroendocrinol 7(6), 475-482 (1995).

55. Tanti, A., Rainer, Q., Minier, F., Surget, A. \& Belzung, C. Differential environmental regulation of neurogenesis along the septotemporal axis of the hippocampus. Neuropharmacology 63(3), 374-384 (2012).

56. O'Leary, O. F. \& Cryan, J. F. A ventral view on antidepressant action: roles for adult hippocampalneurogenesis along the dorsoventral axis. Trends Pharmacol Sci 35(12), 675-687 (2014).

57. Anacker, C. et al. Hippocampal neurogenesis confers stress resilience by inhibiting the ventral dentate gyrus. Nature 559(7712), 98-102 (2018).

58. Tanti, A. \& Belzung, C. Neurogenesis along the septo-temporal axis of the hippocampus: are depression and the action of antidepressants region-specific? Neuroscience 252, 234-252 (2013).

\section{Acknowledgements}

This work was supported by research grant 3-13563 (MOST China-Israel cooperation (2016YFE0130500) from the State of Israel Ministry of Science, Technology, \& Space to GR-L and CX, by research grant 3-14356 from the State of Israel Ministry of Science, Technology, \& Space to GR-L and by a DFG grant STO 488/6-1 to OS and G.R.-L.

\section{Author Contributions}

A.Z. initiated the project. A.Z., G.R.-L., O.S. and H.V. developed the project. A.Z. and G.R.-L. planned and A.Z. and R.L.A. performed the behavioral and biochemical experiments. X.L., C.X., H.V., O.S., R.L.A., G.R.-L. and A.Z. had equal contribution in discussing the results and writing the manuscript.

\section{Additional Information}

Competing Interests: The authors declare no competing interests.

Publisher's note Springer Nature remains neutral with regard to jurisdictional claims in published maps and institutional affiliations. 
(c) (i) Open Access This article is licensed under a Creative Commons Attribution 4.0 International License, which permits use, sharing, adaptation, distribution and reproduction in any medium or format, as long as you give appropriate credit to the original author(s) and the source, provide a link to the Creative Commons license, and indicate if changes were made. The images or other third party material in this article are included in the article's Creative Commons license, unless indicated otherwise in a credit line to the material. If material is not included in the article's Creative Commons license and your intended use is not permitted by statutory regulation or exceeds the permitted use, you will need to obtain permission directly from the copyright holder. To view a copy of this license, visit http://creativecommons.org/licenses/by/4.0/.

(C) The Author(s) 2019 